\title{
The teaching of multi-disciplinary cancer care: A flipped classroom approach.
}

Helen Sarah Winter, Tessa Greenhalgh, Naomi Warner, Ashvina Segaran, Prof Ashok Handa

Background: Multi-disciplinary team (MDT) cancer care was introduced to improve cancer outcomes in UK. Teaching of MDTs involves observation from the "back of the room". A flipped classroom approach was used as a tool for experiential learning of participation in a meeting. Team dynamics, shared decision-making and representing patient views are important in MDTs.

Methods: Four true-to -life cancer cases requiring a multi-modality approach were developed. A flipped classroom was developed following feedback from students. Participants were given cases; individual roles with speciality-specific information; links to cancer resources and guidelines for case discussions. Following preparation facilitators set the scene and supported the groups to run the case discussion. Observers gave feedback on the decision-making, team dynamics and how the patient's views were voiced. Participants gave feedback on their participation within the team, reflections on their role, and their learning of cancer care.

Results: Eighty 4th year medical students each participated in two cases, with on average of 10 participants per case. Students compared their learning with their observational experiences of attending MDTs. Students rated this experience highly as a learning experience. Attendance was high, although not all students had prepared their roles. Feedback on the sessions was positive with the majority of students preferring this as a method for learning about MDTs. Results included reflections on how it felt be to be a member of the team with a different opinion, how group dynamics affected decision-making and suggestions for improvements for the flipped classroom approach.

Conclusions: A flipped classroom approach to teaching cancer management was rated highly by students. This approach offers a flexible, learning tool that stimulates knowledge application and conceptual understanding. Other professional skills were developed by chairing, presenting evidence from prior preparation and considering the patient's wishes and values. The evaluation of a new innovative way to teach cancer care was well-supported by the students who overwhelmingly have advised to implement the pilot fully into the undergraduate clinical course 